\title{
Prevalence and Risk Factor of Venous Thromboembolism in Korean Patients with Congestive Heart Failure
}

\author{
Moon Ju Jang, ${ }^{1}$ Hyuck-Moon Kwon, ${ }^{2}$ Myung-Ho Jeong, ${ }^{3}$ Doyeun $\mathrm{Oh}^{1 *}$ \\ 'Department of Internal Medicine, CHA University School of Medicine, Seongnam, Korea \\ 2Department of Internal Medicine, College of Medicine, Yeonsei University, Seoul, Korea \\ ${ }^{3}$ The Heart Research Center of Chonnam National University Hospital, Gwangju, Korea
}

Received: 9 June 2018

Revised: 30 July 2018

Accepted: 8 August 2018

*Corresponding author: Doyeun Oh, MD, PhD Department of Internal Medicine, CHA University School of Medicine, 59 Yatap-ro, Bundang-gu,Seongnam 13496, Korea

Tel : +82-31-780-5217

Fax : +82-31-780-5221

E-mail: doh@cha.ac.kr

Copyright (C) Korean Society on Thrombosis and Hemostasis. All rights reserved.
Purpose: Congestive heart failure (CHF) is a major risk factor for venous thromboembolism (VTE) in Western countries. However, its contribution to VTE risk has not been established in an Asian population. To address this issue, we conducted a population-based study and a retrospective observation study to assess the prevalence and risk factors of VTE in Korean CHF patients.

Methods: This population-based study used the Korean Health Insurance Review and Assessment Service database. Patients diagnosed with CHF and VTE between 2007 and 2011 were retrospectively identified by the Korean Classification of Disease. Also, a retrospective observation study was conducted at three teaching hospitals in Korea between 2006 and 2010.

Results: The VTE prevalence in Korean CHF patients was $0.855 \%$ and steadily increased between 2007 and 2011. In the retrospective observation study, 32 CHF patients with VTE, among a total of 1,759 CHF patients, were assessed. Multivariate analyses demonstrated that advanced age ( $\geq 65$ years), recent surgery or trauma, and cancer were associated with VTE in CHF patients.

Conclusion: Our study showed a lower VTE rate in Korean CHF patients than has been previously reported in Caucasians. Elderly age, recent surgery or trauma, and cancer are independent VTE risk factors in Korean CHF patients.

Keywords: Venous thromboembolism, Congestive heart failure, Prevalence, Risk factor

\section{Introduction}

Venous thromboembolism (VTE), including deep vein thrombosis (DVT) and pulmonary embolism (PE), is a major health concern and cause of mortality worldwide. ${ }^{1-4}$ Among the well-established risk factors for VTE, congestive heart failure (CHF) is a well-known major risk factor, especially in hospitalized patients. ${ }^{5-8}$ In CHF patients, the risk of VTE is increased because of multi-factorial causes including blood stasis due to decreased ejection fraction (EF), immobilization, endothelial dysfunction, and hemostatic abnormalities. Thus, VTE is approximately three times more likely to develop in CHF patients than in individuals without CHF.'

Most previous studies regarding VTE in CHF patients were conducted in Caucasian populations. The reported incidence of VTE in CHF patients has widely ranged from $1.63 \%$ to $59 \% .^{5,9-11}$ Recently, several epidemiologic studies in the Asian population demonstrated that VTE incidence is rapidly increasing, making VTE an important health concern in Asian countries., ${ }^{212-16}$ In this respect, we hypothesized that CHF is an important risk factor for VTE in Asian populations. To date, however, despite the clinical significance of CHF as a likely VTE risk factor, its contribution to VTE risk has not been established in an Asian population. Therefore, we conducted this nationwide epidemiologic study using the Korean Health Insurance Review and Assessment Service (HIRA) database to analyze the VTE prevalence in CHF patients. We also performed a retrospective observation study to investigate potential VTE risks in Korean CHF patients.

\section{Methods}

The National Health Insurance (NHI) program of Korea is a compulsory social insurance system that covers the whole population. A government-affiliated organization, HIRA, has designed an accurate claims-review and quality-assessment system for the NHI. These systems made it possible for us to perform a population-based study, because the NHI basically covers the ethnically homogeneous Korean population. The features of the HIRA database have been reported elsewhere in details. ${ }^{12,17}$ All CHF cases were identified with diagnostic codes according to the Korean Classification of Disease, fifth edition (KCD-5) from 2007 to 2011. The CHF diagnostic codes included I50.0 (congestive heart failure), I50.1 (left ventricular failure), and I50.9 (heart failure, unspecified). The DVT codes included I80.2 (DVT, not otherwise specified [NOS]) and I80.3 (embolism or thrombosis of lower extremity, NOS). The codes for PE included I26 (pulmonary thromboembolism), I26.0 (pulmonary embolism with mention of acute cor pulmonale), and I26.9 (pulmonary embolism, NOS). The number of CHF patients and the rates of DVT and PE in CHF patients were evaluated by age, gender, and year of enrollment in the study.

A retrospective observation study was conducted at three teaching hospitals in Korea. Patients $\geq 15$ years of age with a diagnosis of CHF between January 2006 and December 2010 were selected through the KCD-5 codes from the medical database. During this study period, the total number of diagnosed CHF patients was 2,667. After reviewing the medical records of all CHF patients, we excluded $908=$ because of incomplete clinical data. Among the remaining 
1,759 CHF patients, we selected 32 with an objective diagnosis of VTE. In these cases, VTE had been confirmed by venography or compression ultrasonography for DVT and by ventilation-perfusion (V/Q) lung scans or computed tomography (CT) for PE. For each patient, demographic information (age, sex, and body mass index $[\mathrm{BMI}]$ ), comorbid conditions (diabetes mellitus [DM], hypertension [HTN], coronary artery disease [CAD], chronic obstructive pulmonary disease $[\mathrm{COPD}]$, and chronic renal failure $[\mathrm{CRF}]$ ), and VTE risk factors (recent surgery [ $<3$ months], recent trauma/fracture $[<3$ months], immobilization [ $<7$ days], and malignancy) were collected from the medical records. Institutional Review Board approval was obtained from the participating institutions.

Prevalence was estimated as the number of VTE per $100 \mathrm{CHF}$ patients, and 95\% confidence intervals (CI) were determined. Differences between the group of CHF patients with VTE and CHF patients without VTE were assessed using Student's t test. Categorical variables were compared using the chi-square test. Logistic regression analyses were performed to select significant risk factors for VTE among the clinical variables. Multivariate analysis was performed to select independent risk factors for VTE among those clinical variables using logistic regression analysis. All values were calculated as relative risk (RR) or odds ratios (OR) and the corresponding 95\% CI. Significance was defined as $P<0.05$ for all anal- yses. Statistical analyses were conducted using SPSS 19.0 (IBM, NY, USA).

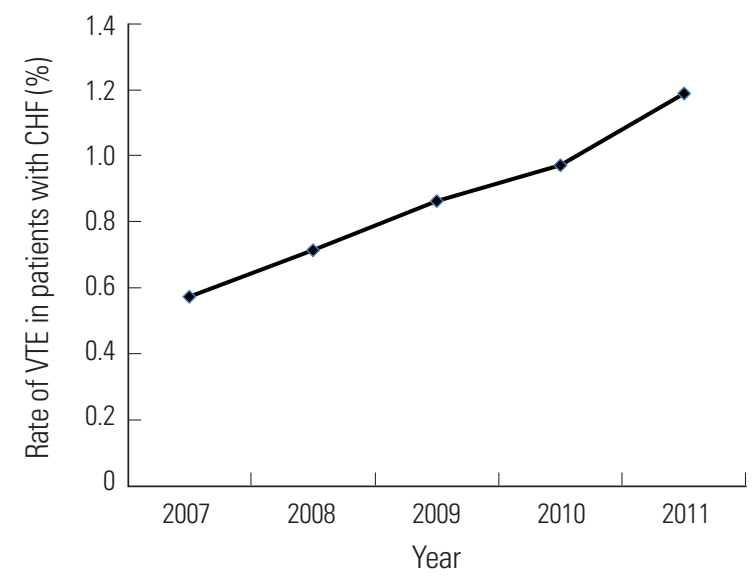

Fig. 1. Rate of VTE in CHF patients by calendar year from 2007 to 2011. The VTE rate was $0.573 \%$ in $2007,0.713 \%$ in $2008,0.862$ in $2009,0.971 \%$ in 2010 , and $1.188 \%$ in 2011 .

Table 1. Prevalence of VTE in Korean CHF patients according to age and gender

\begin{tabular}{|c|c|c|c|c|c|c|c|c|c|}
\hline \multirow{2}{*}{$\begin{array}{l}\text { Age group } \\
\text { (yr) }\end{array}$} & \multicolumn{3}{|c|}{ Male } & \multicolumn{3}{|c|}{ Female } & \multicolumn{3}{|c|}{ Total } \\
\hline & CHF & $\begin{array}{c}\text { CHF with } \\
\text { VTE }\end{array}$ & $\begin{array}{c}\text { Rate, } \\
\%(95 \% \text { CI) }\end{array}$ & CHF & $\begin{array}{c}\text { CHF with } \\
\text { VTE }\end{array}$ & $\begin{array}{c}\text { Rate, } \\
\%(95 \% \text { CI) }\end{array}$ & $\mathrm{CHF}$ & $\begin{array}{l}\text { CHF with } \\
\text { VTE }\end{array}$ & $\begin{array}{c}\text { Rate, } \\
\%(95 \% \text { CI) }\end{array}$ \\
\hline$<40$ & 37,662 & 270 & 0.717 (0.634-0.808) & 27,503 & 155 & $0.564(0.478-0.660)$ & 65,165 & 425 & $0.652(0.592-0.717)$ \\
\hline $40-59$ & 295,949 & 1,181 & 0.399 (0.377-0.422) & 290,930 & 1,295 & $0.445(0.421-0.470)$ & 586,879 & 2,476 & $0.422(0.405-0.439)$ \\
\hline $60-79$ & 493,577 & 4,656 & $0.943(0.916-0.971)$ & 882,992 & 8,983 & 1.017 (0.996-1.039) & $1,376,569$ & 13,639 & 0.991 (0.974-1.008) \\
\hline$>80$ & 97,245 & 1,234 & 1.269 (1.199-1.342) & 298,348 & 2,963 & 0.993 (0.958-1.030) & 395,593 & 4,197 & 1.061 (1.029-1.094) \\
\hline All ages & 924,433 & 7,341 & 0.794 (0.776-0.812) & $1,499,773$ & 13,396 & 0.893 (0.878-0.908) & $2,424,206$ & 20,737 & $0.855(0.844-0.867)$ \\
\hline
\end{tabular}

CHF, congestive heart failure; VTE, venous thromboembolism.

Table 2. Characteristics of VTE in CHF patients

\begin{tabular}{|c|c|c|c|c|c|}
\hline Variables & CHF with VTE, n (\%) & CHF without VTE, n (\%) & $\mathrm{RR}$ & $95 \% \mathrm{Cl}$ & $P$-value \\
\hline Age, yr & 74.48 (9.54) & $70.90(14.02)$ & & & 0.143 \\
\hline Obesity (BMI $\geq 25$ ) & $9(28.12)$ & 493 (28.55) & 0.985 & 0.563 to 1.723 & 0.958 \\
\hline \multicolumn{6}{|l|}{ Comorbid conditions } \\
\hline $\mathrm{DM}$ & $8(25)$ & 536 (31.04) & 0.805 & 0.440 to 1.474 & 0.483 \\
\hline Smoker & $7(21.87)$ & $469(27.1)$ & 0.805 & 0.417 to 1.557 & 0.520 \\
\hline CAD & $6(18.75)$ & $362(20.96)$ & 0.894 & 0.432 to 1.851 & 0.764 \\
\hline COPD & $3(9.37)$ & $97(5.62)$ & 1.669 & 0.559 to 4.987 & 0.359 \\
\hline CRF & $2(6.25)$ & $155(8.97)$ & 0.696 & 0.180 to 2.687 & 0.599 \\
\hline \multicolumn{6}{|l|}{ VTE risk factors } \\
\hline
\end{tabular}

CHF, congestive heart failure; VTE, venous thromboembolism; BMI, body mass index; DM, diabetes mellitus; HTN, hypertension; CAD, coronary artery disease; $\mathrm{COPD}$, chronic obstructive pulmonary disease; CRF, chronic renal failure; OP, operation. 


\section{Results}

During the 5 years (2007-2011) of this study, we identified annually from 444,247 to 500,940 CHF patients using the HIRA database. The rate of VTE in patients with CHF was $0.855 \%$ (95\% CI, 0.8440.867 , Table 1). Except for patients under 40 years of age, the VTE rate increased steadily with advancing age, from $0.652 \%(95 \% \mathrm{CI}$, $0.592-0.717)$ in patients under 40 years of age, $0.422 \%(95 \% \mathrm{CI}$, $0.405-0.439)$ at $40-59$ years of age, $0.991 \%(95 \% \mathrm{CI}, 0.974-1.008)$ at $60-79$ years of age, up to $1.061 \%(95 \% \mathrm{CI}, 1.029-1.094)$ in patients over 80 years of age. The annual VTE rate in patients with CHF was $0.573 \%$ (95\% CI, 0.552-0.594) in 2007, $0.713 \%$ (95\% CI, 0.690 $0.737)$ in $2008,0.862(95 \%$ CI, 0.836-0.889) in 2009, 0.971\% (95\% CI, 0.944-0.998) in 2010, and $1.188 \%$ (95\% CI, 1.156-1.221) in 2011. The rate of VTE increased steadily from 2007 to 2011 (Fig. 1).

We compared the clinical characteristics of $\mathrm{CHF}$ patients according to VTE occurrence (Table 2). From the retrospective observation study, we identified $32 \mathrm{CHF}$ with VTE patients and 1,727 CHFonly patients. Compared with CHF-only patients, CHF patients with VTE had higher proportions of recent surgery or trauma (RR 3.787, 95\% CI 2.026-7.081), immobilization (RR 1.548, 95\% CI 1.0632.254), and cancer (RR 2.901, 95\% CI 1.266-6.648). However, there was no significant association between VTE occurrence and age, gender, BMI, or any comorbid condition (DM, HTN, CAD, COPD, and $\mathrm{CRF}$ ).

Multivariate analysis was performed to investigate independent risk factors for VTE in CHF patients (Table 3). After adjusting for clinical variables, age older than 65 years was independently associated with VTE risk in CHF patients (OR 3.412, 95\% CI 1.009-11.538). In addition, recent surgery/trauma (OR 4.227, 95\% CI 1.765-10.122) and cancer (OR 2.950, 95\% CI, 1.083-8.036) were associated with a significantly increased VTE risk in these patients. However, none of the comorbid conditions investigated, including DM, HTN, CAD, smoking history, COPD, and CRF, was associated with increased VTE risk.

Table 3. Risk factors for VTE in CHF patients

\begin{tabular}{lccc}
\hline Variables & OR & 95\% Cl & P-value \\
\hline Elderly age $(\geq 65$ yr) & 3.412 & 1.009 to 11.538 & 0.048 \\
Gender, male & 1.213 & 0.563 to 2.617 & 0.622 \\
Obesity (BMI $\geq 25)$ & 1.308 & 0.576 to 2.968 & 0.521 \\
Comorbid conditions & & & \\
DM & 0.725 & 0.310 to 1.692 & 0.457 \\
HTN & 0.858 & 0.404 to 1.821 & 0.690 \\
CAD & 0.919 & 0.366 to 2.307 & 0.858 \\
Smoker & 0.725 & 0.287 to 1.830 & 0.496 \\
COPD & 1.710 & 0.491 to 5.960 & 0.400 \\
CRF & 0.631 & 0.141 to 2.824 & 0.547 \\
VTE risk factors & & & \\
$\quad$ OP/Trauma & 4.227 & 1.765 to 10.122 & 0.001 \\
Immobilization & 1.590 & 0.751 to 3.366 & 0.226 \\
Cancer & 2.950 & 1.083 to 8.036 & 0.034 \\
\hline
\end{tabular}

VTE, venous thromboembolism; BMI, body mass index; DM, diabetes mellitus; HTN, hypertension; CAD, coronary artery disease; COPD, chronic obstructive pulmonary disease; $\mathrm{CRF}$, chronic renal failure; $\mathrm{OP}$, operation.

\section{Discussion}

The present study is the first population-based study on VTE prevalence in Asian patients with CHF. In this study, VTE rate in Korean CHF patients was $0.855 \%$. Thus, we demonstrated that the VTE rate in the Korean CHF patient population is lower than in Caucasians with CHF. Interestingly, we found a rapidly increasing VTE rate in $\mathrm{CHF}$ patients in recent years. Additionally, we found that elderly age, recent surgery or trauma, and cancer are independent VTE risk factors in CHF patients. Our study might be valuable for design of relevant healthcare guidelines for Asians in view of the increasing VTE prevalence.

Previous epidemiologic studies reported a wide range of VTE incidences in $\mathrm{CHF}$ patients that ranged from $1.63 \%$ to $59 \%$, depending on the nature of the study. ${ }^{5,6,9-11,18-21}$ In the majority of studies, however, it was reported that VTE incidence in CHF patients was approximately $10 \%$. In the subgroup analysis of the Medical Patients with Enoxaparin trial, Alikhan et al. reported that $14.6 \%$ of CHF patients were diagnosed with DVT. ${ }^{18}$ Randomized controlled studies showed $4.96 \%$ and $12.2 \%$ VTE incidence in hospitalized patients with $\mathrm{CHF}^{22,23}$ From a prospective observational study in a population with severe CHF, Darze et al. ${ }^{19}$ reported that $9.1 \%$ patients were complicated with PE. Our study showed a much lower rate of VTE in Korean CHF patients compared with the reported incidence in Caucasian populations. Ethnicity-dependent VTE epidemiology may be explained by differential genetic predispositions between Asian and Caucasian populations. Different environmental factors and life styles may also contribute to varied VTE epidemiology according to ethnicity. However, a population-based study using the National Hospital Discharge Survey (NHDS) of the United States reported only a $1.63 \%$ VTE rate in American CHF patients. ${ }^{5}$ Comparison of our data with the NHDS data is reasonable because these studies had similar study designs, in which mainly symptomatic VTE cases were selected. Compared with the NHDS data, our study suggests that the VTE rate in CHF patients is lower in Asian populations than in Caucasian populations. Currently, limited data is available on the VTE incidences in Asian CHF patients. To our knowledge, only one Japanese study was published on DVT incidence in CHF patients. ${ }^{24}$ That prospective observational study reported that $11.2 \%$ of CHF patients (18 of 161 patients) were diagnosed with DVT. However, only three patients presented with DVT complications in the proximal legs. Moreover, there were no patients with symptoms suspicious of VTE or PE during prospective observation in the Japanese study. ${ }^{24}$ Thus, in agreement with our results, the Japanese study also suggests that symptomatic VTE has a relatively lower prevalence in Asian populations than in Caucasian populations. Interestingly, however, we found rapidly increasing VTE rate in Korean CHF patients over the years that we examined. During the study period, there was an approximately 2-fold increase of VTE rate. It should be emphasized that this trend did not show any plateau pattern. Increasing VTE prevalence in Korean CHF patients might be explained by the rapid aging of the Korean population and by increased awareness and identification of VTE by Korean physicians. ${ }^{12}$

Although $\mathrm{CHF}$ is the major risk factor for VTE, other risk factors for VTE in CHF patients are not well-established. There are few studies examining risk factors or clinical predictors of VTE in CHF patients. In a prospective study of severe CHF patients admitted to a coronary care unit, Darze et al. reported that PE risk is prominent in patients with cancer, previous VTE history, and right ventricle ab- 
normality. ${ }^{19}$ In addition, Howell et al. ${ }^{25}$ found that previous VTE history, surgical state, and obesity were strongly associated with risk of developing VTE. In our study, we found that elderly age, recent surgery or trauma, and cancer were independent risk factors for VTE occurrence. Thus, as with other acute medical diseases, traditional risk factors may also have an important role for VTE occurrence in CHF patients. However, in a prospective study of medical intensive care unit patients, Hirsch et al. ${ }^{26}$ reported that there was no difference in age, gender, BMI, cancer, and recent surgery between patients with DVT and those without. Further larger studies are warranted to elucidate these conflicting results. In addition to traditionally recognized risk factors, $\mathrm{CHF}$ severity may also predispose one to VTE. Several studies demonstrated the association between decreased EF and risk of VTE occurrence. ${ }^{18,24,25}$ Howell et al. ${ }^{25}$ reported that VTE risk strikingly increased as EF decreased, especially in patients with an EF $<20 \%$. Similarly, the Medical Patients with Enoxaparin study showed an approximately 2-fold increase in VTE frequency in the New York Heart Association (NYHA) class IV group compared with the NYHA class III group $(21.7 \%$ vs. $12.3 \%) .{ }^{18}$ Although we did not assess the association between $\mathrm{CHF}$ severity and VTE risk in this study, CHF severity is closely related to prolonged hospitalization, immobilization, and invasive procedures. ${ }^{27,28}$ It is plausible that decreased cardiac function may be an important risk factor for VTE occurrence in $\mathrm{CHF}$ patients, regardless of ethnicity or race.

Recently, evidence-based practice guidelines of the 9th edition by the American College of Chest Physicians (ACCP) were published for antithrombotic therapy and prevention of thrombosis in acutelyill hospitalized medical patients. ${ }^{29}$ The ACCP guidelines recommend anticoagulant thromboprophylaxis for CHF patients with VTE risk factors including active cancer, previous VTE, reduced mobility, known thrombophilic condition, or recent trauma and/or surgery. Consistent with the ACCP recommendations, our study demonstrated a higher OR for VTE development in cancer patients and in patients who had experienced recent surgery or trauma. However, because these guidelines are based on data mainly from Caucasian individuals, our findings raise the question of whether Asian CHF patients should receive less aggressive thromboprophylaxis than is standard in Caucasian populations. The guidelines are originally based on findings that the incidence of VTE in CHF without prophylaxis is at least higher than $10 \%$ as calf DVT, $2 \%$ as proximal DVT, and $1 \%$ as clinical PE, indicating moderate risk for VTE prevention. In addition, the guidelines recommend against VTE pro- phylaxis in patients with low risk for VTE prophylaxis in that the incidence of VTE without prophylaxis is lower than $2 \%$ as calf DVT, $0.4 \%$ as proximal DVT, and $0.2 \%$ as clinical PE. ${ }^{30-32}$ Based on our results, Korean CHF patients are categorized between low and moderate risk, while CHF patients in Western populations are categorized in the moderate or high risk group. Therefore, we propose that Asian CHF patients with active cancer or recent trauma and/or surgery may need thromboprophylaxis. Although our study does not give confirmatory evidence for this proposed guideline, our findings do provide basic evidence that highlight the need for further study. Design of evidence-based guidelines for Asian CHF patients requires prospective studies of thromboprophylaxis in $\mathrm{CHF}$ patients.

There are several limitations to this study. First, it is possible that physicians had diagnosed the patients as having VTE by clinical suspicion versus using objective diagnostic methods. However, the likelihood of misdiagnosing the number of VTE events that would suffice to interfere with our findings is very low. Second, it is possible that asymptomatic VTE cases were not captured with this method. Asymptomatic VTE patients who were not suspected by physicians were not included in this study. Therefore, the actual incidence of VTE is likely to be greater than the incidence identified in the study. Third, because the HIRA database provided limited information about the patients, we could not perform a nested case-control study. Nonetheless, we conducted a retrospective study in a relatively large population.

The VTE rate in CHF patients is lower in the Korean population than in Caucasians. However, the VTE rate in Korean CHF patients rapidly increased during the study period of 2007 to 2011. To our knowledge, this is the first national population-based epidemiology study in Asia that suggests an ethnicity effect on VTE rate in CHF patients.

\section{Acknowledgments}

This work was supported by grants from the Korean Ministry of Health and Welfare (NSCR-2012-A102065) and Ministry of Education (2017R1D1A1B03029582).

\section{Conflict of Interests}

The authors declare that they have no conflicts of interest for this study.

\section{References}

1. Ageno W, Squizzato A, Garcia D, Imberti D. Epidemiology and risk factors of venous thromboembolism. Seminars in Thrombosis and Hemostasis 2006;32(7):651-8.

2. Naess IA, Christiansen SC, Romundstad P, Cannegieter SC, Rosendaal FR, Hammerstrom J. Incidence and mortality of venous thrombosis: a population-based study. Journal of Thrombosis and Haemostasis 2007; 5(4):692-9.

3. Silverstein MD, Heit JA, Mohr DN, Petterson TM, O'Fallon WM, Melton LJ 3rd. Trends in the incidence of deep vein thrombosis and pulmonary embolism: a 25-year population-based study. Archives of Internal Medicine 1998;158(6):585-93.

4. Spencer FA, Emery C, Joffe SW, Pacifico L, Lessard D, Reed G, et al. Incidence rates, clinical profile, and outcomes of patients with venous thromboembolism. The Worcester VTE study. Journal of Thrombosis and Throm- bolysis 2009;28(4):401-9.

5. Beemath A, Stein PD, Skaf E, Al Sibae MR, Alesh I. Risk of venous thromboembolism in patients hospitalized with heart failure. The American Journal of Cardiology 2006;98(6):793-5.

6. Dean SM, Abraham W. Venous thromboembolic disease in congestive heart failure. Congest Heart Fail 2010;16(4):164-9.

7. Heit JA, O'Fallon WM, Petterson TM, Lohse CM, Silverstein MD, Mohr DN, et al. Relative impact of risk factors for deep vein thrombosis and pulmonary embolism: a population-based study. Archives of Internal Medicine 2002;162(11):1245-8.

8. Piazza G, Fanikos J, Zayaruzny M, Goldhaber SZ. Venous thromboembolic events in hospitalised medical patients. Thrombosis and Haemostasis 2009; 102(3):505 10.

9. Samama MM. An epidemiologic study of risk factors for deep vein thrombosis in medical outpatients: the Sirius study. Archives of Internal Medicine 2000;160(22):
3415-20.

10. Anderson FA, Jr, Wheeler HB, Goldberg RJ, Hosmer DW, Patwardhan NA, Jovanovic B, et al. A populationbased perspective of the hospital incidence and casefatality rates of deep vein thrombosis and pulmonary embolism. The Worcester DVT Study. Archives of Internal Medicine 1991;151(5):933-8.

11. Cogo A, Bernardi E, Prandoni P, Girolami B, Noventa $\mathrm{F}$, Simioni P, et al. Acquired risk factors for deep-vein thrombosis in symptomatic outpatients. Archives of Internal Medicine 1994;154(2):164-8.

12. Jang MJ, Bang SM, Oh D. Incidence of venous thromboembolism in Korea: from the Health Insurance Review and Assessment Service database. Journal of Thrombosis and Haemostasis 2011;9(1):85-91

13. Lee LH, Gu KQ, Heng D. Deep vein thrombosis is not rare in Asia--the Singapore General Hospital experience. Annals of the Academy of Medicine, Singapore 2002;31(6):761-4.

14. Leizorovicz A, Turpie AG, Cohen AT, Wong L, Yoo 
MC, Dans A. Epidemiology of venous thromboembolism in Asian patients undergoing major orthopedic surgery without thromboprophylaxis. The SMART study Journal of Thrombosis and Haemostasis 2005;3(1): 28 34.

15. Piovella F, Wang CJ, Lu H, Lee K, Lee LH, Lee WC, et al. Deep-vein thrombosis rates after major orthopedic surgery in Asia. An epidemiological study based on postoperative screening with centrally adjudicated bilateral venography. Journal of Thrombosis and Haemostasis 2005;3(12):2664-70.

16. Sakon M, Maehara Y, Yoshikawa H, Akaza H. Incidence of venous thromboembolism following major abdominal surgery: a multi-center, prospective epidemiological study in Japan. Journal of Thrombosis and Haemostasis 2006;4(3):581-6.

17. Jang MJ, Bang SM, Oh D. Incidence of pregnancy-associated venous thromboembolism in Korea: from the Health Insurance Review and Assessment Service database. Journal of Thrombosis and Haemostasis 2011; 9(12):2519-21.

18. Alikhan R, Cohen AT, Combe S, Samama MM, Desjardins L, Eldor A, et al. Prevention of venous thromboembolism in medical patients with enoxaparin: a subgroup analysis of the MEDENOX study. Blood Coagulation \& Fibrinolysis 2003;14(4):341-6.

19. Darze ES, Latado AL, Guimaraes AG, Guedes RA, Santos AB, de Moura SS, et al. Incidence and clinical predictors of pulmonary embolism in severe heart failure patients admitted to a coronary care unit. Chest 2005;128(4):2576-80

20. Dries DL, Rosenberg YD, Waclawiw MA, Domanski
MJ. Ejection fraction and risk of thromboembolic events in patients with systolic dysfunction and sinus rhythm evidence for gender differences in the studies of left ventricular dysfunction trials. Journal of the American College of Cardiology 1997;29(5):1074-80.

21. Dunkman WB, Johnson GR, Carson PE, Bhat G, Farrell L, Cohn JN. Incidence of thromboembolic events in congestive heart failure. The V-HeFT VA Cooperative Studies Group. Circulation 1993;87(6 Suppl):VI94 101.

22. Cohen AT, Davidson BL, Gallus AS, Lassen MR, Prin MH, Tomkowski W, et al. Efficacy and safety of fondaparinux for the prevention of venous thromboembolism in older acute medical patients: randomised placebo controlled trial. BMJ 2006;332(7537):325-9.

23. Leizorovicz A, Cohen AT, Turpie AG, Olsson CG, Vaitkus PT, Goldhaber SZ. Randomized, placebo-controlled trial of dalteparin for the prevention of venous thromboembolism in acutely ill medical patients. Circulation 2004;110(7):874-9.

24. Ota S, Yamada N, Tsuji A, Ishikura K, Nakamura M, Ito $\mathrm{M}$. Incidence and clinical predictors of deep vein thrombosis in patients hospitalized with heart failure in Japan. Circulation Journal 2009;73(8):1513-7.

25. Howell MD, Geraci JM, Knowlton AA. Congestive heart failure and outpatient risk of venous thromboembolism: a retrospective, case-control study. Journal of Clinical Epidemiology 2001;54(8):810-6.

26. Hirsch DR, Ingenito EP, Goldhaber SZ. Prevalence of deep venous thrombosis among patients in medical intensive care. JAMA 1995;274(4):335-7.

27. Formiga F, Chivite D, Manito N, Mestre AR, Llopis F,
Pujol R. Admission characteristics predicting longer length of stay among elderly patients hospitalized for decompensated heart failure. European journal of in ternal medicine 2008;19(3):198-202.

28. Philbin EF, Rocco TA Jr, Lynch LJ, Rogers VA, Jenkins P. Predictors and determinants of hospital length of stay in congestive heart failure in ten community hospitals. The Journal of Heart and Lung Transplantation: the official publication of the International Society for Heart Transplantation 1997;16(5):548-55.

29. Kahn SR, Lim W, Dunn AS, Cushman M, Dentali F, Akl EA, et al. Prevention of VTE in nonsurgical patients: Antithrombotic Therapy and Prevention of Thrombosis, 9th ed: American College of Chest Physicians Evidence-Based Clinical Practice Guidelines. Chest 2012; 141(2 Suppl):e195S-226S.

30. Geerts WH, Bergqvist D, Pineo GF, Heit JA, Samama CM, Lassen MR, et al. Prevention of venous thromboembolism: American College of Chest Physicians Evidence-Based Clinical Practice Guidelines (8th Edition). Chest 2008;133(6 Suppl):381S-453S.

31. Geerts WH, Pineo GF, Heit JA, Bergqvist D, Lassen $\mathrm{MR}$, Colwell CW, et al. Prevention of venous thromboembolism: the Seventh ACCP Conference on Antithrombotic and Thrombolytic Therapy. Chest 2004;126 (3 Suppl):338S-400S

32. Nicolaides AN, Breddin HK, Fareed J, Goldhaber S, Haas S, Hull R, et al. Prevention of venous thromboembolism. International Consensus Statement. Guidelines compiled in accordance with the scientific evidence. Int Angiol 2001;20(1):1-37. 